\title{
New metallic mesh designing with high electromagnetic shielding
}

\author{
Longjia Qiu ${ }^{1,2,}$, Li Li ${ }^{1,2}$, Zhifeng Pan $^{1,2}$, Bin Zhu ${ }^{1,2}$, Xiangbing Sun ${ }^{1,2}$, and Wei $\mathrm{Yan}^{1,2}$ \\ ${ }^{1}$ Electro-Optical Equipment Research Institute of AVIC, Luoyang,471025, China \\ ${ }^{2}$ Science and Technology on Electro-optic Control Laboratory, China
}

\begin{abstract}
Metallic mesh is an important technic approach to realize the stealth of the electro-optical radar. This paper demonstrates the designing thoughts of metallic mesh, and in the conclusion, this paper achieves the integrated design method by analysing electromagnetic shielding, permeability and so on. By using this method, a metallic mesh designing with high electromagnetic shielding is designed, which will make the aircraft gain better stealth effect in combat.
\end{abstract}

\section{Introduction}

The fighter is an important weapon to implement long-range precision strike, and it must have good penetration resistance and survivability. As an important electro-optical detection equipment for aircraft penetration, electro-optical radar is also an important scattering source for aircraft exposed to the outer surface of aircraft, so it is necessary to take stealth measures to reduce its RCS. The way to realize the stealth optical window is usually three kinds: one is to use the absorbing material in the structure surface of the optical window, the other is to change the shape design of the optical window structure, and the third is to adopt the metallic mesh to the optical window to shield the radar wave from the optical window.

The metallic mesh can shield the electromagnetic waves from the optical window, because of its submillimetre scale cycle and micron magnitude line width, it shows different diffraction characteristics when the electromagnetic waves at different wavelengths are incident. In the microwave band, the electromagnetic wavelength is longer than the mesh cycle, the metallic mesh at this time is the sub wavelength structure, and the posterior diffraction plays the leading role, most of the electromagnetic waves are reflected, the metallic mesh has electromagnetic shielding characteristics; In the infrared and visible wavelengths, the electromagnetic wave is less than the mesh cycle, the metallic mesh at this time is the macroscopic structure, the forward diffraction plays a leading role, most of the electromagnetic wave transmission to the metallic mesh presents light properties. When a metallic mesh with a high frequency filtering function of electromagnetic wave is loaded on the optical window, the optical window is still transparent to the visible and infrared light, but for the length of the microwave and the radio wave, the optical window is equivalent to the metallization process, which realizes the isolation of electromagnetic waves inside and

\footnotetext{
*Corresponding author: cubitcubit@126.com
} 
outside the optical window. To prevent external electromagnetic interference and internal electromagnetic leakage, especially when the optical window with metallic mesh is loaded on the aircraft with shape conformal, the strong reflection cavity effect for optical window on the radar band can be avoid, which achieve a significant reduction of radar signature signal.

The main advantages of metallic mesh frequency filtering technology for optical window electromagnetic shielding are: by selecting the appropriate mesh cycle and linewidth, and controlling the resonant frequency of the electromagnetic wave, a wide-band electromagnetic shielding with a wavelength greater than millimetre wave can be achieved, and the millimetre wave can be passed through but only shielded from the microwave band [1], and the same control may be achieved to the light transmission band. At the same time through the visible and infrared bands, can also only through the visible band and shielding infrared band [2].

\section{Design technology for stealthy optical window with metallic mesh}

\subsection{Analysis of electromagnetic shielding characteristics for metallic mesh}

When the electromagnetic field that changes over time is exposed to the surface of the conductive body, in which the induced current will be produced, this induced current will produce a new electromagnetic field, the new electromagnetic field is in the opposite direction with and the original one, and offset the original electromagnetic field changes, this phenomenon is Faraday electromagnetic induction phenomenon. It indicates that the conductive shielding body has the ability of attenuation the electromagnetic wave intensity [3].

The equivalent circuit method is proposed by Ulrich to analyse the photoelectric properties of metallic mesh, a metallic mesh film can be equivalent to the circuit which composed by electrical impedance (inductance, capacitance, resistance). It indicates that the metallic mesh is a highly efficient and transparent conductive film [4], as shown in figure 1.
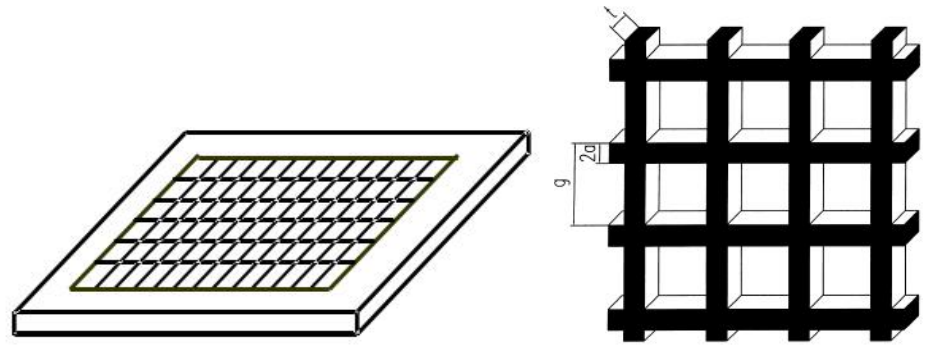

Fig. 1. Metallic mesh diagram.

In this paper, the integral equation is constructed by using the metallic mesh film with periodic array element, the spectral domain Galerkin method is used as the basic analysis method, the transmission coefficient and the reflection coefficient of the metallic mesh are calculated by the moment method, and the electromagnetic shielding efficiency is obtained. Because of the surface resistance of metallic mesh, the spectral domain equation of the ideal conductor is modified, and the theoretical analysis and experimental study are carried out on this basis [5].

In free space, when the thickness of the metallic mesh is much smaller than the line width, and the line width is much smaller than the line cycle, when $\mathbf{T}<<\mathbf{2 a}, \mathbf{2} \mathbf{a}<<\mathbf{g}$, the 
shielding effect for the vertical incident radar waves, metallic mesh has normalized admittance:

$$
y=f_{0}\left[\ln \left(\sin \frac{\pi a}{g}\right)\right]\left(\frac{f}{f_{0}}-\frac{f_{0}}{f}\right)^{-1}
$$

In equation $(1), \mathrm{f}_{0}$ is normalized resonant frequency.

Because the mesh cycle $\mathrm{g}$ is approximately equal to the wavelength of the resonant wave, $\mathbf{f}_{\mathbf{0}} \approx 1, \mathbf{f}=\mathbf{g} / \lambda, \lambda$ is the wavelength of radar signal. At present, the frequency range of radar signal is from $0.05 \sim 40 \mathrm{GHz}$, the range of frequency domain is widely. Generally, the commonly used radar signal wavelength is $16.7 \mathrm{~mm} \sim 150 \mathrm{~mm}$, the mesh period studied in this paper is $200 \mu \mathrm{m} \sim 400 \mu \mathrm{m}$, so when $\lambda>>\mathbf{g}$, the normalized admittance is approximately:

$$
y \approx-\frac{g}{\lambda}\left[\ln \left(\sin \frac{\pi a}{g}\right)\right]
$$

The transmittance of the metallic mesh with vertical incident wave is:

$$
T(0,0)=\frac{4 y^{2}}{1+4 y^{2}}
$$

For $2 \mathrm{GHz} \sim 18 \mathrm{GHz}$ Radar bands, $\mathrm{y}^{2} \ll 1$, the equation (3) can be approximated as:

$$
T(0,0) \approx \frac{4 g^{2}}{\lambda^{2}}\left[\ln \left(\sin \frac{\pi a}{g}\right)\right]^{2}
$$

The shielding factor $(\mathrm{DB})$ is:

$$
S=-10 \log _{10} T
$$

From the above we can see that:

- Electromagnetic shielding effectiveness decreases with cycle $\mathbf{g}$ and increases with line width $\mathbf{2 a}$;

- The metallic mesh cycle $\mathbf{g}$ has a significant effect on the electromagnetic shielding coefficient, and the effect of line width $\mathbf{2 a}$ is less.

\subsection{Analysis of the light transmittance characteristics for the metallic mesh}

In the optical frequency range, the metallic mesh effects as a diffraction grating, producing multistage diffraction for the incident light, and the transmittance of the mesh is equivalent to the sum of the total transmittance of each diffraction sequence, which can be approximated as [6]:

$$
T_{\text {total }}=\frac{(\mathrm{g}-2 \mathrm{a})^{2}}{g^{2}}
$$

For IR imaging applications, only the level 0 of the diffraction sequence is effective, while the other levels have less influence on imaging than the level 0 , which can be negligible. It is usually possible to use scalar diffraction theory to analyze the attenuation of optical system transmittance, while the point diffusion function applied to metallic mesh can be expressed by the square modulus of the pupil function with Fourier transform, which 
ignoring the influence of the optical path difference, and the transmittance for the pupil function of the optical system is as follows:

$$
t(x, y)=\left[\operatorname{rect}\left(\frac{x}{g-2 a}, \frac{x}{g-2 a}\right) * * \sum_{m} \sum_{n} \delta(x-n g, y-m g)\right] \times \operatorname{rect}\left(\frac{x}{N g}, \frac{x}{N g}\right)
$$

In equation (7):

- $\quad * *$ represents two-D convolution;

- $\quad \mathbf{N}$ is the number of mesh irradiated by the incident light;

- $\mathbf{n}, \mathbf{m}$ is integer sequence $(\ldots,-2,-1,0,1,2, \ldots)$. ) ;

- $\quad \operatorname{rect}(\mathbf{x}, \mathbf{y})$ is two-D rectangular transmittance function.

If the diffraction kernel area of the metallic mesh is assumed to be a circle which area is $\mathbf{( N g ) ^ { 2 }}$, then the radiation distribution of the point diffusion function for the optical system of metallic mesh is as follows:

$$
I\left(\theta_{x}, \theta_{y}\right)=\frac{(g-2 a) 4}{g 4}\left\{\begin{array}{l}
\sum \sum \sin c^{2}\left[\frac{n(g-2 a)}{g}\right] \times \sin c^{2}\left[\frac{m(g-2 a)}{g}\right] \sin c^{2}\left[\frac{N g}{\lambda}\left(\theta_{x}-\frac{n \lambda}{g}\right)\right] \\
\times \sin c^{2}\left[\frac{N g}{\lambda}\left(\theta_{y}-\frac{m \lambda}{g}\right]\right.
\end{array}\right\}
$$

Use $\boldsymbol{\theta}_{\mathbf{x}}, \boldsymbol{\theta}_{\mathbf{y}}$ replace the cosine angle of the diffraction light with the $\mathbf{x}, \mathbf{y}$ axes, $\boldsymbol{\lambda}$ is wavelength:

$$
\sin c(x)=\frac{\sin (\pi x)}{\pi x}
$$

When $\theta_{x}=\frac{n \lambda}{g}, \theta_{y}=\frac{m \lambda}{g}$

The peak value of the complex diffraction sequence generated by the incident light through the metallic mesh is equal to the peak value of the point diffusion function of the optical system without the mesh, then any sequence of the diffraction sequence, it takes the ratio of the energy for the occupied point diffusion function is:

$$
\frac{E(n, m)}{E_{p s f}}=\frac{(g-2 a)^{4}}{g^{4}} \sin c^{2}\left[\frac{n(g-2 a)}{g}\right] \sin c^{2}\left[\frac{m(g-2 a)}{g}\right]
$$

With metallic mesh, the energy ratio of the center level 0 is:

$$
T_{0}=\frac{E_{(0,0)}}{E}=\left(\frac{(g-2 a)^{2}}{g^{2}}\right)^{2}
$$

The duty ratio of metallic mesh is in square relationship with $T_{\text {total }}=\frac{(\mathrm{g}-2 \mathrm{a})^{2}}{g^{2}}$.

From the above deduction we can see that:

- The transmittance increases with the cycle $\mathbf{g}$, and decreases with the line width 2a increase;

- The cycle $\mathbf{g}$ has a greater impact on the transmittance, and the line width $\mathbf{2 a}$ effect is less.

\section{Design of a new high electromagnetic shielding metallic mesh}




\subsection{Design of metallic mesh with different configurations}

In order to meet the requirements of using, while maximizing the stealth effect of the aircraft, the first thing in metallic mesh designing is to determine the transmittance to meet the requirements of indicators, on this basis, as far as possible to obtain a higher electromagnetic shielding efficiency.

Based on the typical square (figure 2), hexagonal (figure 3) and circular metallic mesh (figure 4), this paper presents a new type of metallic mesh (figure 5), which improves the efficiency of electromagnetic shielding under the condition of keeping the light transmittance unchanged. In this paper, the comparative analysis of the metallic mesh configuration is shown as follows:

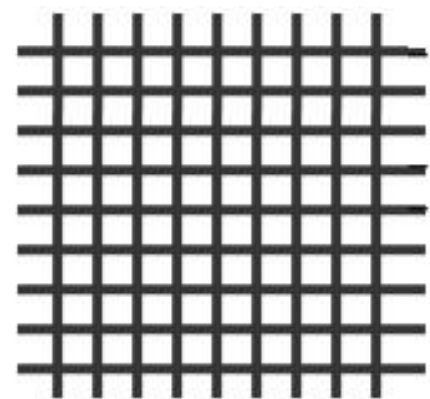

Fig. 2. Square metallic mesh diagram.

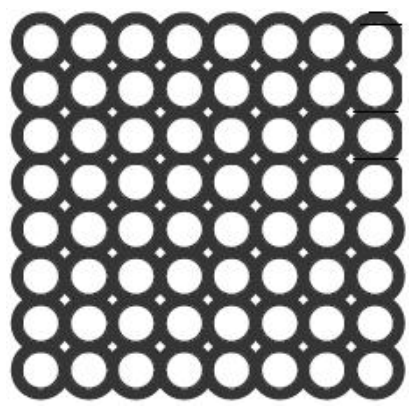

Fig. 4. Circular metallic mesh diagram.

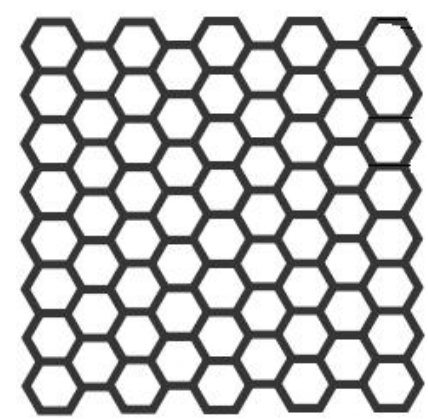

Fig. 3. Hexagonal metallic mesh diagram.

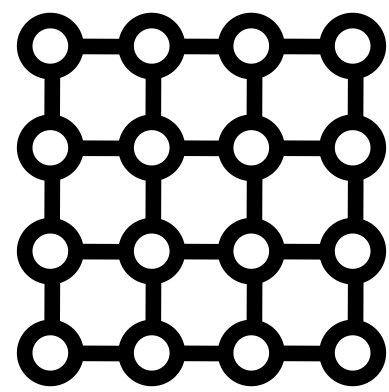

Fig. 5. A new metallic mesh diagram.

\subsection{Settings of simulation condition}

Electromagnetic shielding simulation conditions are setting as follows:

- Optical material: plain glass;

- Optical swatch size: $99 \mathrm{~mm} \times 87 \mathrm{~mm} \times 12 \mathrm{~mm}$;

- Radar wave frequency: $1 \mathrm{GHz} 18 \mathrm{GHz}$.

\subsection{Simulation results of electromagnetic shielding effectiveness for different configurations of metallic mesh}

According to the above simulation condition, and adjusting the metallic mesh cycle, the infrared transmittance of each configuration metallic mesh is equivalent, and the shielding performance is simulated and compared, the results are shown in the following table. 
Table 1. Comparison of simulation results for electromagnetic shielding effectiveness with different configurations of metallic mesh.

\begin{tabular}{|c|c|c|c|c|c|}
\hline Configuration & Transmittance & $\begin{array}{c}\text { Level 0 } \\
\text { Transmittance }\end{array}$ & $\begin{array}{l}\text { Shielding } \\
\text { Mean DB }\end{array}$ & $\begin{array}{c}\text { Shielding } \\
\text { Maximum } \\
\text { db }\end{array}$ & $\begin{array}{c}\text { Shielding } \\
\text { Minimum } \\
\text { db }\end{array}$ \\
\hline Square & $\mathrm{T}_{\text {square }}$ & $\mathrm{T}_{\text {square}-0}$ & $\mathrm{~S}_{\text {square-mean }}$ & $\mathrm{S}_{\text {square-max }}$ & $\mathrm{S}_{\text {square-min }}$ \\
\hline Hexagonal & $\mathrm{T}_{\text {square }}$ & $\mathrm{T}_{\text {square }-0}$ & $\underset{\text { mean }}{\mathrm{S}_{\text {square- }}+0.535}$ & $\begin{array}{c}\mathrm{S}_{\text {square- }} \\
\max +0.331\end{array}$ & $\begin{array}{c}\mathrm{S}_{\text {square- }} \\
\min +0.627\end{array}$ \\
\hline Circular & $\mathrm{T}_{\text {square }}$ & $\mathrm{T}_{\text {square}}-0$ & $\begin{array}{c}\mathrm{S}_{\text {square-mean }}{ }^{-} \\
1.455\end{array}$ & $\begin{array}{c}\mathrm{S}_{\text {square-max }} \\
2.102\end{array}$ & $\begin{array}{c}\mathrm{S}_{\text {square-min }}{ }^{-} \\
1.594\end{array}$ \\
\hline $\begin{array}{c}\text { New } \\
\text { configuration }\end{array}$ & $\mathrm{T}_{\text {square }}$ & $\mathrm{T}_{\text {square}}-0$ & $\begin{array}{c}\mathrm{S}_{\text {square- }} \\
\text { mean } \\
+0.858\end{array}$ & $\begin{array}{c}\mathrm{S}_{\text {square- }} \\
\max +0.298\end{array}$ & $\begin{array}{c}\mathrm{S}_{\text {square- }} \\
\min +0.654\end{array}$ \\
\hline
\end{tabular}

It is shown from the above table that the electromagnetic shielding efficiency is further improved by the new type metallic mesh with the equivalent infrared transmission.

\section{Summary}

In summary, it can be found that the changes of metallic mesh cycle and line width parameters, the metallic mesh electromagnetic shielding efficiency and the impact of infrared transmittance is more significant, and the metallic mesh configuration changes has less influence for the metallic mesh electromagnetic shielding efficiency and infrared transmittance. In this paper, we proposed a new configuration metallic mesh which has improved the efficiency of electromagnetic shielding compared with other typical metallic mesh, and it has the equivalent infrared transmittance with others. This new metallic mesh can make the aircraft gain better stealth effect in combat.

\section{References}

1. Analysis and design of transparent conductive coating and filters. KOHIN M, WEIN S J, TRAYLOR J D, et al. Optical Engineering. (1993)

2. Infrared thin films. SHIMSHOCK R P. Proceedings of SPIE the International Society for Optical Engineering. (1991)

3. Feng Xiaoguo, Fang Liang, Sun Lianchun. Design and fabrication of metal grating film Structure [J]. Optics and precision Engineering, (2005), 13(1): pp 59-64.

4. Gao Jinsong, Sun Lianchun, Zheng Xuanming . etc. Infrared transparent conductive metal grating film [J]. Optics Technology, (2001), 27(6): pp 558-59.

5. Shen Zhenfeng, Feng Xiaoguo. Electrical inductive mesh film for electromagnetic shielding of infrared windows [J]. IR technology, (2008), 8(30): pp 468-71.

6. Fan Zhigang. Photoelectric test Technology [M]. Beijing electronic industry press, (2004). 